\title{
El enfoque de la lesson study en el practicum del máster de musicoterapia con mujeres víc- timas de violencia de género
}

\section{The lesson study approach in the practicum of the master's music therapy with women vic- tims of gender violence}

\author{
Empar Guerrero Valverde \\ Universidad Católica San Vicente Mártir (UCV), \\ Valencia (España) \\ empar.guerrero@ucv.es
}

Fecha de recepción 11-04-2018

Fecha de aceptación 22-05-2018

Guerrero Valverde, E. (2018). El enfoque de la lesson study en el practicum del máster de musicoterapia con mujeres víctimas de violencia de género. Revista Prácticum, 3(1), 49-61. 
El enfoque de la lesson study en el practicum del máster de musicoterapia con mujeres víctimas de violencia de género

\section{Resumen}

Este trabajo describe la experiencia formativa e investigadora, llevada a cabo, con una alumna del Máster de Musicoterapia de la Universidad Católica "San Vicente Mártir" en su período de prácticas curriculares en el que diseñó, implementó y evaluó, un programa musicoterapéutico dirigido a un grupo de mujeres víctimas de violencia de género de la Asociación Alanna de Valencia, supervisado por la tutora de prácticas que a su vez era la tutora académica. Todo ello se realizó siguiendo las fases de las Lesson Study, adaptándolas al ámbito y al contexto de intervención.

Esta experiencia se realizó a lo largo de curso académico 2017-2018, desde febrero hasta mayo, con un total de 150 horas de prácticas. Con esta propuesta se puso en marcha un proceso formativo en el que se conjugó la intervención directa con las mujeres y la investigación sobre la práctica realizada por la alumna y la tutora profesional.

El objetivo de este trabajo es demostrar que las Lesson Study pueden también implementarse en un ámbito diferente al escolar y que ofrece nuevas posibilidades en el proceso de aprendizaje del alumnado en prácticas, en nuestro caso, del Máster de Musicoterapia.

\section{Abstract}

This paper described the formative and research experience carried out with a student of the Music Therapy Master of the Catholic University "San Vicente Mártir" in her period of curricular practices in which designed, implemented and evaluated a music therapy program directed to a group of women victims of gender violence of the Alanna Association of Valencia, supervised by the internship tutor who was also the academic tutor. All this was done following the phases of the Lesson Study, adapting them to the scope and intervention context.

This experience was carried out throughout the 2017-2018 academic year, from February to May, with a total of 150 hours of practice. With this proposal, a training process was launched direct intervention with women and research on the practice carried out by the student and the professional tutor.

The objective of this paper is to demonstrate that the Lesson Study can also be implemented in a different field to the school and that it offers new possibilities in the learning process of the students in practices, in our case, of the Master of Music Therapy. 


\section{Palabras clave}

Practicum, Lesson Study, posgrado, investigación, musicoterapia

\section{Keywords}

Practicum, Lesson Study, postgraduate, research, music therapy

\section{Introducción}

Las Lesson Study (LS) son un proceso de acción para el cambio e investigación que facilita la comprensión de la realidad sobre la que se investiga (Soto y Pérez, 2015) y que persigue mejorar la práctica educativa siendo el eje de la formación Inicial en algunos países de Asia y de Europa.

Algunos autores (Stenhouse, 1975; Kemmis 1988 y Elliot, 2015) planteaban la necesi dad de incorporar la investigación a la práctica docente, superando "la distancia entre los que planifican y los que aplican, entre el desarrollo profesional de los docentes y la experimentación curricular" (Stenhouse, 1975 citado en Soto y Pérez, 2015 , p. 18). Las LS se han introducido como respuesta a esa necesidad y gracias a su sistematización y estructura permiten alcanzar los objetivos planteados en la investigación/acción.

Para implementar adecuadamente la metodología de las LS, deben desarrollarse las siguientes fases, tal y como describen Soto y Pérez (2015, p. 20-22)

1. Definir el problema, "debatir y formular en grupo los objetivos del aprendizaje a largo plazo de los estudiantes y su relación con la propuesta concreta que queremos trabajar partiendo de nuestras propias prácticas y experiencias" (p. 20).

2. Diseñar cooperativamente una «lección experimental». Se "diseña conjuntamente un plan de acción, no solo para producir una propuesta didáctica, sino también para comprender como y porqué este diseño puede funcionar" (p. 21)

3. Enseñar y observar el desarrollo de la propuesta didáctica diseñada previamente. Durante esta fase un miembro del equipo pone en práctica en su aula el plan diseñado por el grupo, mientras el resto del equipo escucha, observa y registra evidencias sobre el aprendizaje de los estudiantes en relación a los objetivos propuestos (p. 21) 
El enfoque de la lesson study en el practicum del máster de musicoterapia con mujeres víctimas de violencia de género

4. Recoger las evidencias y discutir su significado. "Se trata de poner en común, contrastar, evaluar y reflexionar sobre las observaciones registradas, y utilizarlas para comprender la potencialidad pedagógica de la propuesta inicial y proponer su mejora y reformulación" (p. 22).

5. Analizar y revisar la propuesta. "Como consecuencia del debate, se pueden introducir mejoras en el plan de acción y, en su caso, en el guion de seguimiento y observación del mismo. Un nuevo proceso creativo de diseño a la luz de nuevas aportaciones propias y ajenas constituye el eje de esta fase" ( $p$. 22)

6. Desarrollar el proyecto revisado en otra clase por otro/a docente y observar de nuevo, "repitiendo el proceso de observación, análisis y mejora de la propuesta revisada por parte del resto del grupo" (p.22)

7. Discutir, evaluar y reflexionar sobre las nuevas evidencias y difundir la experiencia en un contexto ampliado: "Esta fase se ocupa, finalmente, de describir y documentar, analizar y valorar la lección dentro de un grupo más amplio de debate, de modo que otros docentes puedan entender, aprender, utilizar y aprender sobre la experiencia desarrollada" (p. 22).

Las LS adquieren especial relevancia en el S. XX en Japón, y desde finales de este siglo y principios del S. XXI se van introduciendo en nuestra cultura y en nuestro sistema educativo (Soto y Pérez, 2015). Aunque, este enfoque ha sido implementado y evaluado sobre todo en el ámbito educativo con docentes, existen experiencias en las que se ha experimentado en la formación inicial de los estudiantes de magisterio a través del Practicum como en Japón, (Chassels y Melville, 2009), Malasia, China o Suecia, lugar en el que se transforma en Learning Study, y en universidades como la de Stavanger, Semarang y Málaga, entre otras.

El Practicum se concibe como una vía para superar la posible fragmentación del conocimiento adquirido en las diferentes disciplinas favoreciendo la relación entre la teoría aprendida en las aulas y la práctica desarrollada en contextos laborales reales, ofreciendo la posibilidad de guiar al alumnado en su práctica reflexiva para mejorar su práctica profesional.

Actualmente el Practicum es una materia que está presente en la mayor parte de estudios universitarios, desde que se diseñaron los estudios de grado y posgrado, siguiendo las directrices establecidas en el Espacio Europeo de Educación Superior, convirtiéndose en una asignatura troncal de gran significación y relevancia (Latorre y Blanco, 2011) y muy valorada por los estudiantes (Manso y Martín, 2014) considerándola como una asignatura fundamental para su formación académica.

El ejercicio de la práctica profesional, en contextos laborales reales, favorece el desarrollo de habilidades y capacidades relacionadas con la futura profesión. "El Practicum es un periodo formativo-práctico, que responde a una programación curricular, por lo general se desarrolla en centros externos a la institución universitaria, implicando a diferentes colectivos (alumnado, tutores de universidad y de empresa), que trata de integrar la teoría y la práctica en una situación real de actividad profesional, mediante la participación y el aprendizaje activo de los practicantes" (Ventura, 2005, p. 28). La formación práctica se transforma así en un punto clave a la hora de generar experiencias formativas integradoras (González, y Méndez, 2017). 
El conocimiento que se adquiere vendrá determinado por diferentes factores relacionados entre sí, como son, las características de los centros en los que se desarrolla el Practicum, con las aptitudes y actitudes del tutor profesional y académico y con los planteamientos pedagógicos que se establecen desde la universidad.

Todo ello vendrá determinado por la orientación pedagógica que adquiere esta materia y por el modelo de aprendizaje en el que se enmarca. Schön (1992) sugería tres modos de entender el Practicum:

a) desde una concepción técnica en la que el conocimiento profesional se entiende en términos de hechos, reglas y procedimientos que deben ser aplicados en la práctica;

b) desde una concepción basada en las reglas para el desempeño de una profesión en la que el alumnado aprende a razonar acerca del camino a seguir en situaciones problemáticas;

c) desde una concepción de reflexión en la acción en la que se aprende a reflexionar desde la práctica, aprendiendo a reconocer e identificar situaciones problemáticas sobre las que encontrar nuevas formas de actuar. Esta última concepción, adquiere un gran significado en la propuesta de las LS ya que éstas sitúan al profesor como investigador.

Asimismo, Zabalza (2011) identifica como Practicum Curricular aquel en el que deben complementarse "los aprendizajes académicos (teóricos y prácticos) con la experiencia (también formativa, es decir vinculada a aprendizajes) en centros de trabajo" (p. 104) y considera que el Practicum que se halle integrado en los programas formativos debe cumplir cinco funciones (Zabalza, 2013). Estas son:

- Aproximar a los estudiantes a escenarios profesionales reales.

- "Generar marcos de referencia o esquemas cognitivos de forma tal que los aprendizajes académicos queden iluminados por su "sentido" y "naturaleza" en la práctica profesional" (p. 61).

- Llevar a cabo nuevas experiencias formativas (adquirir nuevos conocimientos, desarrollar nuevas habilidades, reforzar o modificar actitudes, etc.).

- Sirven para que el alumnado sea consciente de las actividades que se llevan a cabo en su futuro ámbito laboral y "tomar consciencia de las propias lagunas formativas" (p. 63).

- Ofrecer la oportunidad de vivir sobre los propios escenarios profesionales, sus dinámicas, la naturaleza de las intervenciones que se llevan a cabo en ellos, el sentido que los profesionales dan a su trabajo, etc. 
Estas funciones deben desarrollarse en los centros de prácticas y son los tutores profesionales los que adquieren un papel relevante en el proceso formativo ya que deberán ofrecer el marco apropiado para que el aprendizaje se produzca adecuadamente, creando entornos plurales y diversos para enriquecer la experiencia. Esto es posible si conocen qué es lo que se espera de ellos, qué es lo que el alumnado debe aprender y qué es lo que pueden y deben enseñar.

Para que el Practicum sea considerado un espacio de aprendizaje, debe situar al alumnado en el eje principal del proceso y guiarlo para "aprender cómo aprender y cómo investigar sintetizando, asimilando y generando contenidos fuera de la propia especialidad, así como presentando soluciones creativas dentro de situaciones ambiguas y complejas. Aprender a cooperar, escuchar, debatir, dialogar y contrastar con perspectivas diferentes e incluso discrepantes" (Soto y Pérez, 2015, p. 18), siendo todo ello objeto también de las LS.

Por todo ello se considera que el Practicum es una materia adecuada para aplicar los planteamientos de las LS, entendiendo que en el fondo de este enfoque está el trabajo colaborativo y cíclico de una lección, entendiéndola como un proceso de interacción entre docentes y alumnado y que puede tener lugar durante un periodo de tiempo prolongado (Elliot, 2015). Las LS son consideradas una herramienta poderosa para favorecer la reconstrucción del conocimiento práctico (Pérez, Soto y Servan, 2015), respetando la orientación de la reflexión-acción de Schön.

\section{El Practicum en el Máster de Musicoterapia}

El Máster de Musicoterapia, con 60 ECTS, se implementó en la Universidad Católica "San Vicente Mártir" en el año 2006, en la Facultad de Psicología y surge con el objetivo de formar profesionales que actúen como agentes de salud preparados para poder trabajar, a nivel preventivo y/o terapéutico, en ámbitos sanitarios, educativos y sociales.

El Prácticum, con un total de 9 ECTS, tiene como objetivo integrar al alumnado en un contexto de aprendizaje ubicado en campos reales relacionados con la práctica clínica y con el desempeño del rol profesional del musicoterapeuta. Para ello, desarrollará un conjunto de intervenciones de evaluación, prevención o intervención, planificando y desarrollando uno o más procesos de intervención en Musicoterapia.

Las competencias que debe adquirir el estudiante son: capacidad de razonamiento crítico y creativo; capacidad de comunicación no-verbal, verbal y escrita; saber aplicar la musicoterapia en las distintas fases del proceso terapéutico en un individuo o grupo, según el ámbito o campo de aplicación; recopilar la experiencia de las sesiones clínicas y aportar sus primeras intervenciones como musicoterapeutas.

El alumnado en prácticas estará supervisado en todo momento por un tutor académico o tutor interno (profesor de la universidad), quien llevará a cabo un conjunto de actuaciones a lo largo del período de prácticas que garanticen la calidad del proceso formativo y por un tutor externo (profesional del centro laboral) que ofrecerá al alumnado, prácticas formativas que le ayuden a integrar conocimientos prácticos, aplicándolos a situaciones reales. 
Las prácticas de Musicoterapia, con una duración de 150 horas, pueden llevarse a cabo en diferentes ámbitos: desde hospitales a asociaciones que desarrollan programas para personas con autismo u otro tipo de discapacidad, en centros educativos, con niños y niñas con necesidades educativas específicas, o en el ámbito sociocomunitario con diferentes colectivos, entre los que se encuentra el de mujeres víctimas de violencia de género $\left(\mathrm{VVG}^{\circ}\right)$.

\section{Contextualización de las Prácticas en las que se implementó las Lesson Study adaptadas al contexto y al ámbito de intervención}

En el curso académico 2016-2017 se puso en marcha la III edición del Taller de Musicoterapia con 10 mujeres VVGo que participaron de manera voluntaria en la actividad y que fueron derivadas desde la Asociación Alanna ubicada en Valencia. Este Taller lo viene desarrollando desde el 2014 una Musicoterapeuta, (tutora profesional), que a su vez es profesora en el Postgrado de Musicoterapia y por tanto es también tutora académica de la alumna en prácticas que se incorporó al taller "Alégrate de ser tu misma". La Musicoterapia se define como "el uso de la música y/o de sus elementos (sonido, ritmo, melodía y armonía) por un terapeuta cualificado con un/una cliente o grupo, en un proceso dirigido a facilitar y promover la comunicación, las relaciones, el aprendizaje, la movilización, la expresión, la organización y otros objetivos que se consideren relevantes; con el fin de suplir necesidades de tipo físico, emocional, mental, social y cognitivo. La musicoterapia tiene como finalidad desarrollar potenciales y/o restaurar funciones del individuo de forma que él o ella puedan conseguir una integración tanto intra como interpersonal $y$, como consecuencia, una mejor calidad de vida a través de la prevención, la rehabilitación o el tratamiento" (WFMT, 1996).

El Método de trabajo que se utiliza con este grupo es el Abordaje Plurimodal en Musicoterapia (APM) (Schapira, 2007) ya que considera al ser humano como una unidad biopsicosocialespiritual, concibe a cada usuario/a de manera independiente (determinismo psíquico), tiene en cuenta la transferencia y contratransferencia que puede emerger entre paciente y musicoterapeuta, y, por último, incorpora la idea de la música interna como aquella que habita en el inconsciente de cada persona.

Este método contempla la posibilidad de utilizar cuatro técnicas diferentes y complementarias, siendo el musicoterapeuta el que decide cuál es el más adecuado de usar en un determinado momento y con una determinada población. Estos ejes de acción son: Improvisación musical terapéutica; Trabajo con canciones; Estimulación a través de imágenes y sonido (EISS); Uso selectivo de la música editada (Hugo, Ferrari y Schapira, 2007).

La intervención Musicoterapéutica se llevó a cabo desde febrero hasta mayo con un total de 18 sesiones de una hora y media cada una. Las dos primeras sesiones sirvieron para conocer el grupo y determinar los objetivos tanto del Practicum de la alumna como de la intervención. Las dos últimas fueron de evaluación musicoterapéutica.

Todas las sesiones mantenían la misma estructura: acogida y bienvenida (en la que cada mujer escribía el objetivo sobre el que quería centrarse en la sesión), actividad musicoterapéutica (utilizando alguna de las cuatro técnicas descritas anteriormente), puesta en común (en la que compartían lo vivido y experimentado en la sesión) y despedida (se llevaba a cabo una valoración de la sesión). 


\section{La adaptación de Lesson Study en el Practicum de Musicoterapia con Mujeres Víctimas de Violencia de Género}

Aunque tradicionalmente la LS se aplican a estudios de magisterio, por las características de esta metodología, se consideró interesante desarrollar sus fases, como experiencia piloto, en el Máster de Musicoterapia con una alumna en prácticas con un grupo de mujeres VVGo.

Como parte del proceso formativo, además de la intervención directa con las mujeres al finalizar cada sesión se mantenían reuniones con la alumna en las que se le invitaba a investigar sobre la propia práctica favoreciendo la conexión entre los conocimientos adquiridos a nivel teórico en las clases del Máster y la práctica llevada a cabo con las MVVGo. Asimismo se analizaba la participación de las mujeres en cada sesión, siguiendo las fases propuestas por las Lesson Study.

En la primera fase, "definir el problema, debatir y formular en grupo los objetivos", en las prácticas que se presentan del Máster de Musicoterapia, se llevaron a cabo las siguientes acciones:

a) En las dos reuniones iniciales (antes de empezar la intervención directa con las mujeres) la tutora profesional comparte la experiencia musicoterapéutica llevada a cabo a lo largo de los tres últimos años con el fin de acercar a la alumna a la realidad del contexto de prácticas.

b) La alumna define sus objetivos de aprendizaje, preguntándose qué es lo que quiere aprender y qué objetivos quiere conseguir a lo largo del Practicum. En este sentido la alumna planteó los siguientes objetivos:

-Proponer, planificar, implementar y evaluar un proyecto de intervención basada en la musicoterapia como herramienta terapéutica con mujeres víctimas de violencia de género.

-Conocer de qué modo la musicoterapia favorece la mejora de la autoestima, la cohesión de grupo y el empoderamiento de las mujeres que han sufrido violencia de género.

-Tener un espacio donde pueda formarme y desarrollarme como musicoterapeuta, pudiendo poner en prácticas los conocimientos adquiridos durante la formación académica del máster.

-Proporcionar nuevas aportaciones teóricas y prácticas sobre la población de estudio. 
c) Para conocer las características del grupo se seleccionan los instrumentos que nos permitan realizar una evaluación inicial. Los elementos utilizados fueron:

- Ficha Musicoterapéutica (Adaptada de Benenzon): Se trata de un documento donde cada usuaria registra sus preferencias y rechazos musicales (incluyendo sonidos, ruidos, instrumentos, canciones concretas o estilos musicales, etc.). Este instrumento nos permite conocer el ámbito sonoro-musical que tiene el grupo. Sirve para tener un primer contacto con las usuarias y saber cuáles son sus preferencias musicales, puesto que será el elemento fundamental de la intervención.

- Escala de Autoestima de Rosenberg (Echeburúa, 1995). La finalidad de esta escala es evaluar la sensación o sentimiento que una persona tiene sobre sí misma. Se muestra así el vínculo que hay entre la autoimagen real y la ideal. Esta escala de 10 ítems se puntúa de A a D, siendo A Muy de acuerdo y $\mathrm{D}$ Muy en desacuerdo.

- Test de Asertividad de Rathus (s.f.). Este test hace referencia a comportamientos con los cuales las personas quieren expresar sus opiniones, preferencias o negarse ante determinadas coacciones de otros/as. Tiene 30 ítems, puntuados de 1 a 6, siendo: 1 Muy poco característico (de mi personalidad); y 6 , Muy característico (de mi personalidad). La puntuación oscila entre -90 , muy poco asertiva; y +90 , muy asertiva.

Con la información obtenida a través del análisis de los resultados obtenidos se inició la segunda fase, "diseño de una sesión experimental" en la que se planificó una sesión de intervención musicoterapéutica, concretamente, aquí se presenta la sesión número 3. Todo ello se llevó a cabo de forma conjunta entre la tutora profesional y la alumna en prácticas, debatiendo, analizando o planteando dudas. La sesión quedó configurada del modo siguiente: 


\section{Objetivos}

- Ofrecer la música y sus elementos como medio alternativo de la comunicación.

- Favorecer el aumento de la autoestima

\section{Materiales}

- $\quad$ - Aula

- Sillas

- Folios

- Rotuladores

- Colchonetas

\section{Desarrollo de la sesión}

\section{Inicio.}

- Comenzaremos preguntando qué tal la semana.

- Tras esto, se les dirá que escriban, sin comentárselo a ninguna compañera, cuál es el objetivo que se han marcado para el día de hoy y lo meterán en el "cofre de los objetivos de musicoterapia".

Para continuar, dividirán en un folio en 4 . En la primera esquina tendrán que escribir, de manera individual, cuál es el animal que más las representa y por qué. - Del mismo modo lo harán con respecto a los colores, ruidos-sonidos y música. Tras esto lo pondrán en común con el grupo completo.

- A continuación, jugaremos a un bingo musical. Se les darán un folio en blanco en el que deberán realizar una enumeración del 1 al 8. Escucharían trozos de canciones que ellas reconocieron como propias o que se encontraban dentro de sus gustos musicales, debían escribirlas sobre el papel (El cantante, principalmente) y, luego, ver si había acertado, si habían fallado o cuáles eran aquellas que habían dejado en blanco.

\section{Intermedio}

- Escucharían una pista de audio, que contenían tres a su vez, e irían siguiendo diversas instrucciones que, de manera vocal, se les fuesen dando. Éstas fueron: (a) Pista de audio 1.1: Reconoce tu cuerpo, frótate las manos, toca tus brazos, tócate la cara, date un pequeño masaje, mírate al espejo, sonríete; (b) Pista de audio 1.2: Cuando veas a alguna de tus compañeras: salúdala, choca las palmas con ella, dile algo bonito (Ej., que pases un buen día); y, (c) Pista de audio 1.3 (En esta nos colocamos en círculo): bailamos siguiendo el ritmo de la música y copiando los movimientos que entre ellas vayan proponiendo

\section{Final}

Se les propone que, para volver a la calma, nos pondremos de la manera más cómoda y le enseñaremos técnicas de relajación centradas en la respiración.

Como tarea final se les dice que, durante la semana, intenten realizar esta actividad todos los días.

Tabla 1: Diseño de la tercera sesión, 21 de febrero de 2017, Elaboración propia.

Aunque aquí se presenta solo una sesión, cabe destacar que se diseñaron diferentes "sesiones experimentales", siguiendo el mismo procedimiento.

Los objetivos del proyecto se definieron también en esta segunda fase ya que esto permitía diseñar un esbozo de la intervención y entender la globalidad de las sesiones. Los objetivos del proyecto quedaron definidos del modo siguiente: 


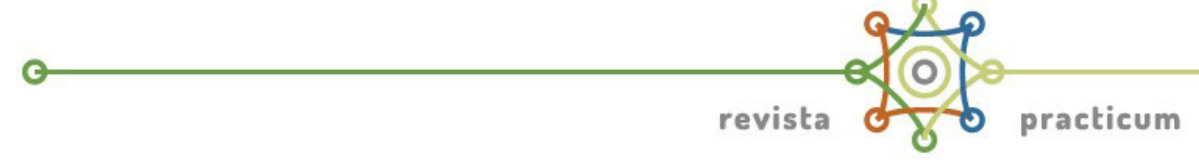

- Ofrecer un espacio donde las usuarias se sientan libres de realizar y comentar todo aquello que crean necesario.

- Ofrecer la música y sus elementos como medio alternativo de la comunicación.

- Favorecer el aumento de la autoestima y autodeterminación de las usuarias, fortaleciendo sus puntos fuertes.

Una vez diseñada la sesión se daba paso a la tercera fase, "enseñar y observar el desarrollo de la propuesta" que consiste en desarrollar la sesión, en este caso, por parte de la alumna en prácticas. La tutora profesional se mantuvo como observadora y tomaba nota de lo que observaba a lo largo de la sesión. Posteriormente, a lo largo del resto de sesiones, los papeles se fueron intercambiando, de modo que se observaba, analizaba y reflexionaba la práctica de ambas.

En algunas sesiones se introdujo la grabación en audio (ya que la imagen no puede ser grabada por la protección de datos) y la fotografía (sin que aparezcan los rostros de las mujeres), con el fin de retomar lo ocurrido en la sesión y profundizar en los aspectos que habían pasado desapercibidos.

Se seleccionaron tres "mujeres-caso" sobre las que se focalizó la observación y a las que se entrevistó al finalizar la sesión con el objetivo de conocer su percepción acerca de lo ocurrido en las dinámicas. Algunas preguntas que se les plantearon fueron: ¿Has disfrutado en la actividad? ¿Para qué te ha servido? ¿Qué es lo que has aprendido? ¿Qué te gustaría aprender más? ¿Qué puede hacer la musicoterapeuta para ayudarte en ese proceso?

En la cuarta fase, "recoger las evidencias y discutir su significado" se evaluaron y analizaron las evidencias recogidas que en nuestro caso fueron las siguientes: la información obtenida mediante la entrevista a las tres "mujeres-caso"; la información recogida por la tutora profesional, mientras la alumna en prácticas desarrollaba la sesión y la escucha del audio grabado a lo largo de la sesión. Algunos de los aspectos analizados fueron:

- La forma en que la alumna se comunica con las mujeres: si las consignas quedaban claras, si la comunicación no verbal era adecuada, si dirigía adecuadamente la actividad y tipo de interacción establecida, entre otros.

- El material utilizado: si era suficiente, si se ajustaba a la necesidad de la actividad, si la música encajaba con los objetivos propuestos, etc.

- Se analizó si se consiguieron o no los objetivos propuestos al inicio de la intervención, incidiendo en las diferencias observadas entre lo planteado al inicio de la intervención y los resultados obtenidos.

- La observación realizada en cada sesión, centrada en las "mujeres-caso" se centró los siguientes aspectos: la participación, la comunicación interpersonal, si tomaban la iniciativa o no en las actividades, si expresaban su opinión o sus sentimientos. Todo esto se valoraba porque formaba parte de los objetivos de la sesión. 
El enfoque de la lesson study en el practicum del máster de musicoterapia con mujeres víctimas de violencia de género

Con toda la información analizada y llevando a cabo una reflexión profunda de lo ocurrido en la sesión se reformularon algunos objetivos, se preparó la siguiente sesión y se seleccionaron otras tres "mujeres-caso" cumpliendo así la fase cinco de las LS, "analizar y revisar la propuesta".

Sucesivamente, a lo largo de toda la intervención, se mantuvieron tanto el análisis como la reflexión de lo ocurrido en cada sesión, atendiendo diferentes aspectos e introduciendo las modificaciones necesarias.

En el enfoque de las LS se habla de una sexta fase "desarrollar el proyecto revisado en otra clase por otro/a docente y observar de nuevo" que en nuestro caso no pudo ser llevada a cabo con un grupo diferente, pero lo que sí se pudo hacer fue, con la propuesta revisada, proponer nuevas sesiones introduciendo mejoras, cambiando objetivos y actividades y seleccionando nuevas "mujeres-caso" (el grupo estaba configurado por diez mujeres). Asimismo, cada sesión era coordinada por la alumna en prácticas o por la musicoterapeuta de forma que se revisaba la práctica profesional de las dos.

La séptima y última fase de las LS, "discutir, evaluar y reflexionar sobre las nuevas evidencias y difundir la experiencia en un contexto ampliado" tuvo lugar en cuatro momentos diferentes:

- Al finalizar cada sesión se iniciaba el proceso de revisión, mejora y análisis y se diseñaba la sesión siguiente.

- En la defensa del TFM que tuvo lugar ante un tribunal especializado, evaluando el trabajo presentado.

- En las IV Jornadas de Diversidad organizadas por Florida Universitària, celebradas en Catarroja (Valencia) mediante la presentación de una comunicación. La Comunicación se compartió con profesionales especializadas en la intervención con mujeres.

- En el III Congreso Iberoamericano de Investigación en Musicoterapia (GIIMT) celebrado en Valencia en el que también se presentó una comunicación con el trabajo realizado y los resultados obtenidos en la intervención. En este evento se pudo intercambiar información con otra Musicoterapeuta que trabaja también con Mujeres VVGo.

\section{Conclusiones}

La aplicación de la metodología de la LS, en el Practicum del Máster de Musicoterapia ha supuesto una gran oportunidad para profundizar en el proceso de aprendizaje de la alumna en prácticas y ha permitido a la tutora profesional introducir mejoras en su práctica profesional ya que también se ha analizado su intervención.

En cuanto a los resultados obtenidos, siguiendo el enfoque de las LS cabe destacar lo siguiente: 
- Con respecto a las mujeres que participaron en el Taller se cumplieron los objetivos planteados en la intervención, ya que al finalizar se volvieron a pasar las dos escalas utilizadas al inicio: Escala de Autoestima de Rosenberg (Echeburúa, 1995) y el Test de Asertividad de Rathus y se observó que el grupo había mejorado tanto en autoestima como en asertividad (estos datos podrán consultarse en breve en las actas del III Congreso Iberoamericano de Investigación en Musicoterapia). Asimismo también se alcanzaron los otros dos objetivos planteados. Todas las participantes coincidieron en que "les había gustado que se les preguntara sobre el proceso que se estaba llevando a cabo, ya que es algo que no suele hacerse".

- Con respecto a la alumna en prácticas se constató que a lo largo del proceso había mejorado, no solo su práctica como musicoterapeuta, sino que había ampliado su conocimiento sobre la intervención con este colectivo de mujeres y había aprendido a diseñar, desarrollar y evaluar un proceso de intervención musicoterapéutico. A todo ello cabe añadir que destacó de forma positiva lo que había supuesto trabajar de forma cooperativa con la tutora profesional y subrayó la importancia de las entrevistas a las mujeres para conocer su percepción sobre la intervención de la que están siendo objeto, ya que se había obtenido información valiosa para reorientar el taller.

A menudo, las prácticas se convierten en una experiencia que favorece la adquisición de nuevos aprendizajes basándose sobre todo en la observación que el alumnado realiza sobre la práctica del profesional. Con este enfoque se favorece un aprendizaje más profundo y consciente, puesto que se da más relevancia a la experiencia, la práctica y la experimentación y esto conlleva nuevas formas de percibir la realidad (Pérez, Soto y Serván, 2015).

En este proceso el tiempo dedicado a diseñar, reflexionar, valorar y reconducir los aspectos mejorables de la práctica ha sido tan importante como el tiempo dedicado a la acción e intervención, lo que supone también un cambio con respecto a lo que es el Practicum, en el que, a veces, se dedica poco tiempo a la reflexión y al análisis de la práctica profesional.

Otro aspecto que cabe destacar es la importancia que han adquirido las "mujeres-caso" en cuanto a la información que han aportado sobre la sesión conociendo sus perspectivas de lo que ha funcionado para ellas, cómo se han sentido, qué aspectos creen que deberían cambiar, entre otros. De este modo, la intervención puede ajustarse mucho más y adaptarse mejor a las necesidades del grupo.

La observación, el análisis y la reflexión sobre todo lo ocurrido en las prácticas, a través de las LS, han ayudado a mejorar la práctica profesional, a cuestionar lo que ya se daba por sabido, a constatar que hay otras formas de plantear el Practicum y a que el enfoque que ofrece las LS puede ser aplicable a otros ámbitos profesionales adaptándolas al contexto, pero sin perder la esencia de las mismas. 
El enfoque de la lesson study en el practicum del máster de musicoterapia con mujeres víctimas de violencia de género

\section{Bibliografia}

Chassels, C. y Melville, W. (2009) Japanese Lesson Study in an Initial Teacher Education Program: Benefits and Challenges. Canadian Journal of Education 32, 4, 734-763 Recuperado de https://files.eric.ed.gov/fulltext/EJ883522.pdf

Elliot, J. (2015). Lesson y learning Study y la idea del docente como investigador. Revista Interuniversitaria de Formación del Profesorado, 84, 29-46

González-Brignardello, M.P., Méndez Zaballos, L. (2017). Desarrollo de un Practicum Virtual: fundamentos y claves. Revista Prácticum, Vol 2(2) 50-68.

Kemmis, S (1988). El Currículum: más allá de la teoría de la reproducción. Madrid. Morata.

Latorre, M.J. y Blanco, F.J. (2011). El Practicum como espacio de aprendizaje profesional para docentes en formación. REDU. Revista de Docencia Universitaria, 9(2), 35-54.

Manso Ayuso, J. \& Martín Ortega, E. (2014). Valoración del Máster de Formación de Profesorado de Educación Secundaria: estudio de casos en dos universidades, Revista de Educación, 364, 145-169

Pérez Gómez, A.I.; Soto Gómez, E. y Serván Núñez, M.J. (2015). Lesson Studies: repensar y re-crear el conocimiento práctico en cooperación. Revista Interuniversitaria de Formación del Profesorado, 83, 81-101

Schön, D. (1992). La formación de profesionales reflexivos. Barcelona. Paidós.

Soto Gómez, E. y Pérez Gómez, A.I. (2015). Lessons Studies: un viaje de ida y vuelta recreando el aprendizaje comprensivo. Revista Interuniversitaria de Formación del Profesorado, $83,15-28$

Stenhouse, (1975). An Introduction to Curriculum Research and Development. London. Heinemann. Educational. Books.

Ventura Blanco, J.J. (2005). El Practicum en los estudios pedagógicos y la i $n \quad$ serción laboral. Nuevos enfoques ante el reto europeo. (Tesis Doctoral). Universidad de Barcelona, Barcelona. Recuperado en: http://tesisenred.net/bitstream/handle/10803/2898/TESIS_JJVENTURA.pdf?sequence $\quad=1$

World Federation of Music Therapy. Recuperado en http://www.musictherapyworld.net/ WFMT/Home.html

Zabalza Beraza, MA. (2001). Formación del profesorado universitario. Revista de investigación educativa, RIE. Vol. 19, No 2, 2001, 659-662

Zabalza Beraza, M.A (2011) El Practicum en la formación universitaria: estado de la cuestión. Revista de Educación, 354, 21-43

Zabalza Beraza, M.A (2013) El Practicum y las Prácticas en empresas en la formación universitaria. Madrid: Narcea 